\title{
TORSION FREE COVERING MODULES
}

\author{
EDGAR ENOCHS
}

Let $A$ be an integral domain and $K$ its field of fractions. An $A$ module $E$ is said to be torsion free if $\alpha x=0$ for $\alpha \in A, x \in E$ implies $\alpha=0$ or $x=0$. We will say that a submodule $E_{1}$ of an $A$-module $E$ is pure in $E$ if $\alpha E_{1}=\alpha E \cap E_{1}$ for all $\alpha \in A$. Then if $E$ is torsion free, a submodule $E_{1}$ of $E$ is pure in $E$ if and only if $E / E_{1}$ is torsion free. Clearly the union of a chain of pure submodules of a module is still a pure submodule and if $E_{2} \subset E_{1}$, are submodules of $E$ such that $E_{2}$ is pure in $E_{1}$ and $E_{1} / E_{2}$ pure in $E / E_{2}$ then $E_{1}$ is pure in $E$.

It is well known that for any $A$-module $E$ there exists a torsion free $A$-module $E_{1}$ and an epimorphism $p: E \rightarrow E_{1}$ such that if $\phi$ is any linear mapping from $E$ into a torsion free module $F$ then there is a unique linear mapping $f: E_{1} \rightarrow F$ such that $f \circ p=\phi$, i.e., the diagram

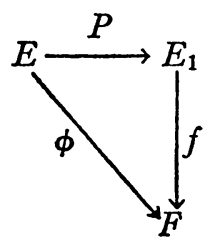

is commutative. It suffices to let $E_{1}$ be $E / E^{\prime}$ where $E^{\prime}$ is the torsion submodule of $E$, i.e., the set of elements of $E$ which are not free and $p$ the canonical mapping $E \rightarrow E / E^{\prime}$.

The object of this paper is to show that for any module $E$ there exists a torsion free $A$-module $T(E)$ and a linear mapping $\psi: T(E) \rightarrow E$ which is unique "up to isomorphism" subject to the two conditions

(1) the kernel of $\psi$ contains no nontrivial pure submodules of $E$,

(2) if $\phi: F \rightarrow E$ is a linear mapping where $F$ is torsion free then there is a linear mapping $f: F \rightarrow T(E)$ such that $\psi \circ f=\phi$.

Such a mapping $\psi$ will be called a torsion free covering of $E$ and $T(E)$ will be called a torsion free covering module of $E$. A linear mapping $\psi: E^{\prime} \rightarrow E$ will be said to have the torsion free factor property if for any linear mapping $\phi: F \rightarrow E$, where $F$ is torsion free there exists a linear mapping $f: F \rightarrow E^{\prime}$ such that $\psi \circ f=\phi$.

We first prove four lemmas.

LEMma 1. If $\psi: E^{\prime} \rightarrow E$ has the torsion free factor property and $E_{1}$ is a submodule of $E$ then the linear mapping $\psi^{-1}\left(E_{1}\right) \rightarrow E_{1}$ which agrees with $\psi$ on $\psi^{-1}\left(E_{1}\right)$ has the torsion free factor property.

Received by the editors September 6, 1962. 
Proof. Trivial.

Lемма 2. If $E$ is injective then $\psi: E^{\prime} \rightarrow E$ has the torsion free factor property if and only if for every linear map $\phi: F \rightarrow E$, where $F$ is torsion free and injective there is a linear mapping $f: F \rightarrow E^{\prime}$ such that $\psi \circ f=\phi$.

Proof. The condition is clearly necessary. If $\phi_{1}: F_{1} \rightarrow E$ is any linear mapping where $F_{1}$ is torsion free, then since $F_{1}$ is a submodule of a torsion free injective (hence divisible) module $F$ and since $E$ is injective there exists a linear mapping $\phi: F \rightarrow E$ such that $\phi \mid F_{1}=\phi_{1}$. Then if $f: F \rightarrow E^{\prime}$ is such that $\psi \circ f=\phi$ then $\psi \circ\left(f \mid E_{1}\right)=\phi_{1}$.

LeMma 3. For every module $E$ there exists a torsion free module $E^{\prime}$ and a linear mapping $\psi: E^{\prime} \rightarrow E$ having the torsion free factor property.

Proof. Using Lemma 1 and the fact that every module is a submodule of an injective module we see that it suffices to assume that $E$ is injective. Then using Lemma 2, we see that in order to prove that a linear mapping $\psi: E^{\prime} \rightarrow E$ has the torsion free factor property it suffices to show that if $\phi: F \rightarrow E$, where $F$ is torsion free and injective then there is a linear mapping $f: F \rightarrow E^{\prime}$ such that $\psi \circ f=\phi$.

If we let $E^{\prime}$ be the direct sum of sufficiently many copies of $K$ then clearly there exists a linear mapping $\psi: E^{\prime} \rightarrow E$ such that for any linear mapping $\phi^{\prime}: K \rightarrow E$ there is a linear mapping $f^{\prime}: K \rightarrow E^{\prime}$ such that $\psi \circ f^{\prime}=\phi^{\prime}$. Then since any torsion free injective module $F$ is the direct sum of a family of submodules isomorphic to $K$, clearly for any linear mapping $\phi: F \rightarrow E$ there is a linear mapping $f: F \rightarrow E^{\prime}$ such that $\psi \circ f=\phi$.

LEMMA 4. If $\psi: E^{\prime} \rightarrow E$ has the torsion free factor property and $N$ is a submodule of $E^{\prime}$ contained in the kernel of $\psi$ then the induced mapping $E^{\prime} / N \rightarrow E$ has the torsion free factor property.

Proof. Trivial.

In particular we see that if $\psi: E^{\prime} \rightarrow E$ has the torsion free factor property where $E^{\prime}$ is torsion free and $N$ is a maximal element among the pure submodules of $E^{\prime}$ contained in the kernel of $\psi$ then the induced mapping $E^{\prime} / N \rightarrow E$ is a torsion free covering of $E$.

This remark coupled with Lemma 3 gives us:

THEOREM 1. Every module $E$ has a torsion free covering.

Now we need to show the uniqueness of the torsion free covering.

THEOREM 2. If $\psi^{\prime}: E^{\prime} \rightarrow E$ and $\psi^{\prime \prime}: E^{\prime \prime} \rightarrow E$ are two torsion free coverings of $E$ and $f: E^{\prime} \rightarrow E^{\prime \prime}$ is a linear mapping such that $\psi^{\prime \prime} \circ f=\psi^{\prime}$ then $f$ is an isomorphism. 
Proof. Since $\psi^{\prime \prime}$ is a torsion free covering of $E$ there exists a linear mapping $f: E^{\prime} \rightarrow E^{\prime \prime}$ such that $\psi^{\prime \prime} \circ f=\psi^{\prime}$. But then the kernel of $f$ is a pure submodule of $E^{\prime}$ (since $E^{\prime \prime}$ is torsion free) which is contained in the kernel of $\psi^{\prime}$. But then since $\psi^{\prime}$ is a torsion free covering, the kernel of $f$ is 0 . Thus $f$ is a monomorphism and so Card $\left(E^{\prime}\right) \leqq$ Card $\left(E^{\prime \prime}\right)$. Similarly Card $\left(E^{\prime \prime}\right) \leqq$ Card $\left(E^{\prime}\right)$ so Card $\left(E^{\prime}\right)=$ Card $\left(E^{\prime \prime}\right)$, i.e., all torsion free coverings of $E$ have the same cardinality. Thus let $X$ be a set containing the elements of $E^{\prime}$ and $E^{\prime \prime}$ and such that Card $(X)$ $>$ Card $\left(E^{\prime}\right)$. Let $\mathcal{F}$ be the set of pairs $\left(E_{0}, \psi_{0}\right)$, where $E_{0}$ is an $A$ module whose elements are elements of $X$ and where $\psi_{0}$ is a linear mapping $E_{0} \rightarrow E$ which is a torsion free covering of $E$. Then $\left(E^{\prime}, \psi^{\prime}\right)$ and $\left(E^{\prime \prime}, \psi^{\prime \prime}\right)$ belong to $\digamma$.

Partially order $\mathcal{F}$ by setting $\left(E_{0}, \psi_{0}\right) \leqq\left(E_{1}, \psi_{1}\right)$ if $E_{0}$ is a submodule of $E_{1}$ and $\psi_{1} \mid E_{0}=\psi_{0}$. Then $\mathcal{F}$ has maximal elements for if $\mathcal{C}$ is a chain of $\mathcal{F}$ let $E^{*}$ be the union of the first coordinates of the pairs in $\mathcal{C}$ with the unique structure of an $A$-module such that $E_{0}$ is a submodule of $E^{*}$ for each $\left(E_{0}, \psi_{0}\right)$ in $\mathcal{C}$ and let $\psi^{*}: E^{*} \rightarrow E$ be the unique linear mapping such that $\psi^{*} \mid E_{0}=\psi_{0}$ for each pair $\left(E_{0}, \psi_{0}\right)$ in $\mathrm{C}$.

Then $\psi^{*}$ clearly has the torsion free factor property. If $N$ is a pure submodule $E^{*}$ contained in the kernel of $\psi^{*}$ then $N \cap E_{0}$ is a pure submodule of $E_{0}$ contained in the kernel of $\psi_{0}$ for each $\left(E_{0}, \psi_{0}\right)$ in $\mathfrak{C}$. Thus $N \cap E_{0}=0$ for each $\left(E_{0}, \psi_{0}\right)$ in $\mathcal{e}$ so $N=0$. Thus $\left(E^{*}, \psi^{*}\right)$ belongs to $\mathcal{F}$. Clearly $\left(E^{*}, \psi^{*}\right)$ is an upper bound of $\mathfrak{C}$.

Thus assume $\left(E^{*}, \psi^{*}\right)$ is a maximal element of $\mathcal{F}$.

Now let $f_{1}: E^{*} \rightarrow E^{\prime}$ be any linear mapping such that $\psi^{\prime} \circ f_{1}=\psi^{*}$. By our previous remarks we know $f_{1}$ is a monomorphism. We would like to show that it is also an epimorphism. Let $Y \subset X$ be such that Card $(Y)=$ Card $\left(E^{\prime}-f_{1}\left(E^{*}\right)\right)$ and such that $E^{*} \cap Y=\varnothing$. Such a $Y$ is available since $\operatorname{Card}(X)>\operatorname{Card}\left(E^{\prime}\right)=\operatorname{Card}\left(E^{*}\right)$. Let $E_{0}=E^{*} \cup Y$ and let $g$ be a bijection $E_{0} \rightarrow E^{\prime}$ such that $g \mid E^{*}=f_{1}$ and $g(Y)=E^{\prime}$ $-f_{1}\left(E^{*}\right)$. Then $E_{0}$ can be made uniquely into an $A$-module so that $g$ becomes an isomorphism. Letting $E_{0}$ denote this module we see that $E^{*}$ is a submodule of $E_{0}$, that $\left(E_{0}, \psi^{\prime} \circ g\right)$ is an element of $\mathcal{F}$ and $\psi^{\prime} \circ g \mid E^{*}=\psi^{\prime} \circ f_{1}=\psi^{*}$ so that $\left(E^{*}, \psi^{*}\right) \leqq\left(E_{0}, \psi^{\prime} \circ g\right)$. But $\left(E^{*}, \psi^{*}\right)$ is a maximal element of $\mathcal{F}$, hence $Y=\varnothing$ so $E^{\prime}-f_{1}\left(E^{*}\right)=\varnothing$ or $f_{1}$ is an epimorphism. Similarly any linear mapping $f_{2}: E^{*} \rightarrow E^{\prime \prime}$ such that $\psi^{\prime \prime} \circ f_{2}=\psi^{*}$ is an epimorphism. But $f \circ f_{1}$ is such a mapping since $\psi^{\prime \prime} \circ f \circ f_{1}=\psi^{\prime} \circ f_{1}=\psi^{*}$ hence $f \circ f_{1}$ is an epimorphism but then $f$ must be an epimorphism. But $f$ is a monomorphism hence an isomorphism. This completes the proof.

Using the fact that the Pontrjagin dual of a compact Abelian group is torsion free if and only if the group is connected [3] we get 
Corollary. Every compact Abelian group $G$ can be embedded uniquely up to isomorphism in a connected compact Abelian group $G^{\prime}$ in such a manner that every continuous homomorphism of $G$ into a connected compact Abelian group can be extended to $G^{\prime}$ and so that $G^{\prime}$ has no closed connected proper subgroups containing $G$.

ThEOREM 3. If $\psi: T(E) \rightarrow E$ is a torsion free covering of $E$ with kernel $G$ then the sequence

$$
0 \rightarrow \operatorname{Ext}_{A}^{n}(F, G) \rightarrow \operatorname{Ext}_{A}^{n}(F, T(E)) \rightarrow \operatorname{Ext}_{A}^{n}(F, E) \rightarrow 0
$$

is exact if $F$ is torsion free and if $n \geqq 1$.

Proof. By definition of $T(E), \operatorname{Hom}(F, T(E)) \rightarrow \operatorname{Hom}(F, E) \rightarrow 0$ is exact whenever $F$ is torsion free. Choose

$$
0 \rightarrow K \rightarrow L \rightarrow F \rightarrow 0
$$

exact with $L$ a free module. Then $\operatorname{Ext}_{A}^{i}(K, \quad)$ is naturally isomorphic to $\operatorname{Ext}_{A}^{i+1}(F, \quad)$ for all $i \geqq 1$. Applying the above remarks to $K$, which is torsion free and using induction we get that

$$
0 \rightarrow \operatorname{Ext}_{A}^{i}(F, G) \rightarrow \operatorname{Ext}_{A}^{i}(F, T(E))
$$

is exact for all $i \geqq 1$. Hence

$$
0 \rightarrow \operatorname{Ext}_{A}^{i}(F, G) \rightarrow \operatorname{Ext}_{A}^{i}(F, T(E)) \rightarrow \operatorname{Ext}_{A}^{i}(F, E) \rightarrow 0
$$

is exact for all $i \geqq 1$.

For an example we show:

Lemma 5. If $A$ is a principal ring and $\pi$ a prime then if $E$ is a torsion free covering module of the $A$-module $A /(\pi)$ then $E$ is isomorphic to the $\pi$-adic numbers.

Proof. It is known that the $\pi$-adic numbers are isomorphic to the inverse limit of the inverse system of $A$-modules defined by the canonical mappings $A /\left(\pi^{n+1}\right) \rightarrow A /\left(\pi^{n}\right), n=1,2, \cdots$. Let $E$ denote this limit and let $\psi: E \rightarrow A /(\pi)$ be the projection mapping. It is easy to see that no nontrivial pure submodules of $E$ are contained in the kernel of $\psi$. Let $\phi: F \rightarrow A /(\pi)$ be any linear mapping where $F$ is torsion free. If $\phi=0$ then let $f: F \rightarrow E$ be the null mapping. Then $\psi \circ f=\phi$. If $\phi \neq 0$ we choose a base $\left(\chi_{\iota}+\pi F\right)_{\iota} \in I$ of the vector space $F / \pi F$ over $A /(\pi)$ such that for one $\iota_{0} \in I, \phi\left(\chi_{t_{0}}\right)=1$ and such that $\phi\left(\chi_{\imath}\right)=0$ for $\iota \neq \iota_{0}$. Then the family $\left(\chi_{\imath}+\pi^{n} F\right)_{\iota} \in I$ forms a base of the $A /\left(\pi^{n}\right)$ module $F / \pi^{n} F$ for first suppose $\sum_{\imath \in I} \alpha_{\imath} \chi_{\iota} \in \pi^{n} F$. Then since 
$\left(\chi_{\imath}+\pi F\right)_{\imath} \in I$ is a base of the $A /(\pi)$-module $F / \pi F$ we see that $\pi$ divides each $\alpha_{\imath}$ and so $\pi$ divides $\sum_{\imath \in I} \alpha_{\imath} \chi_{\imath}$. Since $F$ is torsion free the symbol $\sum_{\imath \in I} \alpha_{\imath} \chi_{\imath} / \pi$ is well defined and we have $\sum_{\imath \in I} \alpha_{\imath} \chi_{\imath} / \pi=\sum_{\imath \in I} \alpha_{\imath} / \pi \chi_{\imath}$ $\in \pi^{n-1} F$. Repeating the argument above we see that $\pi^{n}$ divides $\alpha_{\imath}$ which says the family $\left(\chi_{\imath}+\pi^{n} F\right)_{\iota \in I}$ is free over $A /\left(\pi^{n}\right)$. To prove $\left(\chi_{\imath}+\pi^{n} F\right)_{\imath \in I}$ generates $F / \pi^{n} F$ remark that since $F$ is torsion free the map $\chi \rightarrow \pi^{i} \chi$ of $F$ onto $\pi^{i} F$ is an isomorphism which maps $\pi F$ onto $\pi^{i+1} F$. Thus $F / \pi F$ and $\pi^{i} F / \pi^{i+1} F$ are isomorphic $A$-modules. Thus $\left(\pi^{i} \chi_{\iota}+\pi^{i+1} F\right)_{\iota \in I}$ is a set of generators of $\pi^{i} F / \pi^{i+1} F$ for each $i \geqq 1$. But this clearly implies that for any $\chi \in F$ and $i \geqq 1$ we have $\chi-\sum_{\imath \in I} \alpha_{\imath} \chi_{\imath}$ $\in \pi^{i} F$ for some linear combination $\sum_{\imath \in I} \alpha_{\iota} \chi_{\iota}$ of the $\chi_{\iota}$. It is easy to see that no nontrivial pure submodules of $E$ are contained in the kernel of $\psi$. Let $\phi: F \rightarrow A /(\pi)$ be any linear mapping where $F$ is torsion free. If $\phi=0$ then let $f: F \rightarrow E$ be the null mapping. Then $\psi \circ f=\phi$. If $\phi \neq 0$ we choose a base $\left(\chi_{\imath}+\pi F\right)_{\imath \in I}$ of the vector space $F / \pi F$ over $A /(\pi)$ such that for one $\iota_{0} \in I, \phi\left(\chi_{\iota_{0}}\right)=1$ and such that $\phi\left(\chi_{\imath}\right)=0$ for $\iota \neq \iota_{0}$. [Clearly the family $\left(\chi_{\iota}+\pi^{n} F\right)_{\iota \in I}$ forms a base of the $A /\left(\pi^{n}\right)$ module $\left.F / \pi^{n} F\right]$. Hence there exists a linear mapping $f_{n}: F / \pi^{n} F$ $\rightarrow A /\left(\pi^{n}\right)$ such that $f_{n}\left(\chi_{\iota_{0}}+\pi^{n} F\right)=1+\left(\pi^{n}\right)$ and $f_{n}\left(\chi_{\iota}+\pi^{n} F\right)=0$ if $\iota \neq \iota_{0}$. Passing to the limit we get a mapping $f: F \rightarrow E$ such that $\psi \circ f=\phi$.

In more generality the torsion free covering modules of simple $A$ modules have the following interesting property.

Theorem 4. If $S$ is a simple A-module, $a \subset A$ is the annihilator of $S$ and $\psi: T(S) \rightarrow S$ is a torsion free covering of $S$ then $T(S)$ is a direct summand of any module $F$ containing $T(S)$ such that $a T(S)$ $=a F \cap T(S)$.

Proof. Let $j$ be the mapping $T(S) / a T(S) \rightarrow F / a F$ induced by the canonical injection $T(S) \rightarrow F$. By hypothesis, $j$ will be an injection. Then since $F / a F$ is semi-simple there will be a mapping $\phi_{1}: F / a F \rightarrow S$ such that $\phi_{1} \circ j=\psi$. Thus letting $p$ and $p^{\prime}$ denote the canonical mappings from $T(S)$ into $T(S) / a T(S)$ and from $F$ into $F / a F$ we get that $\psi=\psi_{1} \circ p=\phi_{1} \circ p^{\prime} \circ i$ where $i$ is the canonical injection $T(S) \rightarrow F$. But there exists a linear mapping $f: F \rightarrow T(S)$ such that $\psi \circ f=\phi_{1} \circ p^{\prime}$. Thus $\psi \circ f \circ i=\phi_{1} \circ p^{\prime} \circ i=\psi$ hence $f \circ i$ is an automorphism of $T(S)$ by Theorem 2 so that $i(T(S))=T(S)$ is a direct summand of $F$.

We remark that in case $A$ is a principal ideal domain and $S=A /(\pi)$ then the hypothesis $a T(S)=T(S) \cap a F$ is equivalent to $T(S)$ being a pure submodule of $F$. Then the result of this lemma is a special case of Proposition 2.1, p. 371 of [2]. 
I am grateful to Dr. Johann Sonner whose stimulating ideas interested me in this problem.

\section{REFERENCES}

1. H. Cartan and S. Eilenberg, Homological algebra, Princeton Univ. Press, Princeton, N. J., 1956.

2. D. K. Harrison. On infinite abelian groups, Ann. of Math. (2) 69 (1959), 366391.

3. A. Weil, L'integration dans les groupes topologiques et ses applications, Actualités Sci. Ind. no. 869, Hermann, Paris, 1940.

University of South Carolina 